\title{
Why galaxies care about Type Ia supernovae?
}

\author{
Noelia Jiménez, ${ }^{1}$, Patricia B. Tissera ${ }^{2}$ and Francesca Matteucci ${ }^{3}$ \\ ${ }^{1}$ Institut d'Estudis Espacials de Catalunya (ICE, IEEC/CSIC), E-08193 Bellaterra, Barcelona \\ ${ }^{2}$ Physics Department, Universidad Andres Bello, Av. Republica 220, Santiago, Chile. \\ ${ }^{3}$ Dipartimento di Fisica, Universita' di Trieste, Via G. B. Tiepolo, 11, 34100 Trieste. \\ email: jimenez@ieec.uab.es
}

\begin{abstract}
We implement the Single Degenerate (SD) scenario proposed for Type Ia Supernova (SNIa) progenitors in SPH simulations. We analyse the chemical evolution of bulge-type galaxies together with the observed correlations relating SNIa rates with the characteristics of the host galaxy, such as their SFR. The models reproduce the observed signatures shown by $[\mathrm{O} / \mathrm{Fe}]$ ratios in the Galactic Bulge and the present day SNIa rates. Also, the observed correlation found by Sullivan et al. (2006) between SSFR (specific star formation rate) and the SNIa rate per unit of galaxy mass (SSNIaR), naturally arises. This analysis helps to set more stringent constraints to the galaxy formation models and gives some hint on the progenitor problem.
\end{abstract}

Keywords. galaxies: evolution - galaxies: formation - -galaxies: supernovae -galaxies: numerical simulations

\section{Introduction}

Galaxy formation models make use of sub-grid treatments to include complex physical processes which cannot be numerically resolved, as for example, the SNIa explosions (Kobayashi 2004; Jiménez et al. 2011; Tissera et al. 2013). The still uncertain identity of the SNIa progenitors constitutes a major issue for the models since the regulation of both chemical and energetic feedbacks in the gas dynamics and in the overall stellar evolution is affected. Hence, is of utmost importance to confront the model results with observations to learn about galaxy formation and to test the validity of the adopted hypotheses. The information provided by chemical patterns left by the nucleosynthesis products of the stellar populations can provide stringent constraints for galaxy formation models (Tinsley 1979; Matteucci \& Greggio 1986; Tissera et al. 2014). Additionally, observations relating the SNIa rates of galaxies with the characteristics of the host galaxy as their morphology, colours and SFR can be used as constraints to the progenitors or SNIa (Greggio 2010).

The SD scenario is one of the most popular scenario proposed for explaining the SNIa based on a thermonuclear explosion of a C-O white dwarf exceeding the Chandrasekhar mass through accreting from a non-degenerate companion star, where the mass accretion can assume many configurations. We adopt the SD proposed by Matteucci \& Recchi (2001, from now, MR01), where SNIa originate from a binary systems with one C-O WD and a red (super) giant star. The explosion times correspond to the lifetimes of stars in the mass range $0.8-8 M_{\odot}$. The first system made of two $8 M_{\odot}$ stars explodes after $\sim 3-4 \times 10^{7}$ yrs from the beginning of star formation.

\section{The Simulations}

We use the Tree-PM SPH code GADGET-3 (Springel 2005; Scannapieco et al. 2005, 2006), including star formation, chemical enrichment by supernova feedback, 


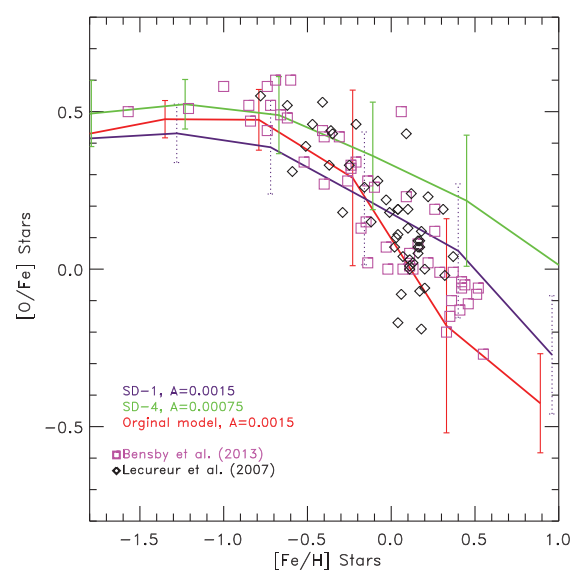

Figure 1. $[\mathrm{O} / \mathrm{Fe}]$ vs. $[\mathrm{Fe} / \mathrm{H}]$ exhibited by the bulge stars in the $\mathrm{SPH}$ simulated galaxies with the SD scenario by Matteucci \& Recchi (2001), with varying A and the Original model. These models are compared to observational $[\mathrm{O} / \mathrm{Fe}]$ ratios for stars in the Galactic Bulge by Bensby et al. (2013) \& Lecureur et al. (2007).

metal-dependent cooling and a multiphase model for the gas component. At the beginning, the gas component represents $\sim 65 \%$ of the total baryonic mass and is distributed in the disc component. The final bulges are formed by a dissipative-dominated component and a central bar concentrated within the inner $\sim 3 \mathrm{kpc}$. The B+D stellar mass are nearly the same for all the simulations $\sim 3.5 \times 10^{10} \mathrm{M}_{\odot}$, typical for a small ellipticals or a bulge of spiral galaxies. Moreover, the analysis of the SFR of the bulges shows that the stars formed during the strong starbursts, where the cold gas is exhausted within $\sim 1$ Gyr. Hence, the new stars formed for which we follow the chemical abundances are mainly part of the bulge component. In the model of Scannapieco et al. $(2005,2006)$ the lifetimes of the binary system that explode as SNIa are assumed to be randomly distributed within a certain range given by $\tau_{S N I a}=[0.1,1]$ Gyr. This model is referred as the "Original model" to distinguish it from the implementation of the SD presented here. Thus, considering a larger minimum lifetime for the distribution of the secondary mass, compared to the predictions of the SD scenario.

\subsection{Calibrating the SD scenario}

The SD scenario involve a free parameter (A), which is associated to the fraction of binary systems of the type necessary to produce SNIa (see MR01 for further details). This value should be adjusted to match the observed SNIa rate at the present time. We use the SNIa rates from spheroidal-dominated galaxies given by Li et al. (2011), obtaining good agreement with models: SD-1 (run with $\mathrm{A}=0.0015$, SNIa rates $=0.002$ $\mathrm{SN} / \mathrm{yr})$ and SD-4 $(\mathrm{A}=0.0075, \mathrm{SNI}$ rates $=0.0016 \mathrm{SN} / \mathrm{yr})$. The Original model $(\mathrm{A}=0.0015$, $\mathrm{SNIa}$ rates $=0.0027 \mathrm{SN} / \mathrm{yr})$ show rates of similar values. We require the models also to reproduce the knee expected in $[\mathrm{O} / \mathrm{Fe}]$ vs $[\mathrm{Fe} / \mathrm{H}]$ distribution. Therefore, we compute the average stellar mass abundance of $[\mathrm{Fe} / \mathrm{H}]$ and $[\mathrm{O} / \mathrm{Fe}]$ for the stars in the bulge of each model and compared to Galactic Bulge stars, taken this comparison as indicative. The sample includes dwarfs and subgiant stars from Bensby et al. (2013) and Red giants stars from Lecureur et al. (2007). Form Fig. 1 it can be seen that the observed data points are best represented by the models SD-4 and SD-1. The former model (SD-4) fits the zero point of the data following the observed trend up to $[\mathrm{Fe} / \mathrm{H}] \sim-0.25$. Meanwhile, SD-1 matches the slope and passes through the data for larger metallicities ranges. These 

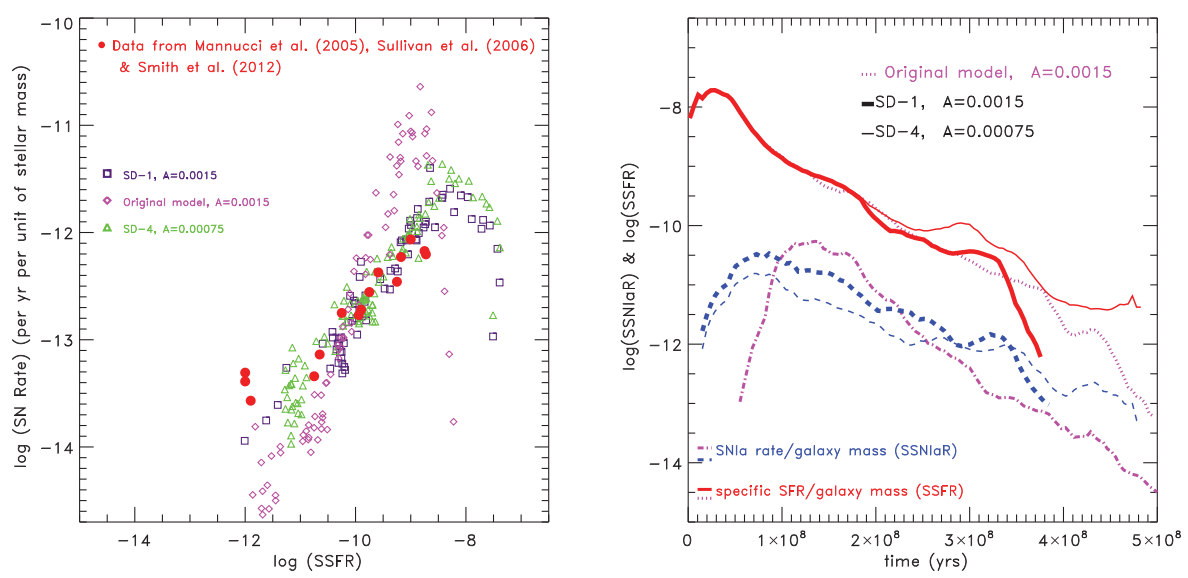

Figure 2. (Left Panel): SSFR as a function of the SSNIaR for all the models. The red circles represent combined data from Sullivan et al.; Mannucci et al.; Smith et al.. Notice that these models also fit the observed $[\mathrm{O} / \mathrm{Fe}]$ ratios coming from the Galactic Bulge and the present-day SNIa rates. (Right Panel): The $\log (\mathrm{SSFR})$ and $\log (\mathrm{SSNIaR})$, evolving with time for all the models.

models predict a long plateau for the $[\mathrm{O} / \mathrm{Fe}]$ ratio and a knee occurring at high $[\mathrm{Fe} / \mathrm{H}]$, as observations suggest. However, at high metallicity the slopes of both models do not follow so nicely the observed $[\mathrm{O} / \mathrm{Fe}]$ ratios. This is perhaps a consequence of yields of Woosley \& Weaver (1995) that do not include mass loss from massive stars. The Original model, albeit simple, fits the data in the whole range.

\section{SNIa rates and SSFR correlation}

By using the SuperNova Legacy Survey (SNLS) galaxy sample Sullivan et al. (2006), showed an existing correlation between the specific SFR and the specific SNIa rates (SSFR and SSNIaR, respectively). He also found this correlation for observations of a morphologically classified sample of galaxies in the local universe presented by Mannucci et al. (2005). Later, Smith et al. (2012) confirmed this correlation, adding the fact that it does not seem to evolve with redshift.

We test if the models can reproduce the observed correlation. For this the SSNIaR and the SSFR are estimated as the galaxy evolves, under the hypothesis that observations might catch galaxies at different stages of evolution (see also Greggio (2010) for a similar approach using analytical models). In the left panel of Fig. 2 we show a combination of the observed data by Smith et al. (2012); Sullivan et al. (2006); Mannucci et al. (2006), and the results for the SD-1, SD-4 and the Original model. Given the reported uncertainties in the zero point of this correlation (see Smith et al. 2012), we focus on the study of the slope of this correlation. Consequently, the simulated relations are normalized by an $a d$ hoc factor to make the comparison of the slope easier. First, we notice that the observed correlation comes out naturally from the SD models. This is remarkable, since it was not used for their calibration. On the other hand, the Original model, albeit reproducing the SNIa rates and the $[\mathrm{O} / \mathrm{Fe}]$ ratios, does not follow the trend displayed by the data, showing a different slope.

All the models show that the SSNIaR increases abruptly with high values of SSFR before reaching the expected observed trend. To understand this behaviour we plot in the right panel of Fig. 2 the logarithm of SSFR and the SSNIaR as a function of time. Here it can be seen that the sharp increase of SSNIaR for high SSFR reflects the onset of 
SNIa and how quickly it reaches the maximum value. The Original model differs from the SD ones showing a delayed peak with a stronger declination after reaching the maximum value. For all the models, after the maximum is reached, both the SSNIaR and the SSFR decrease establishing the observed correlation. For very low SSFR, there is still a residual SSNIaR, as the generation of these events is delayed in time and the correlation is lost.

Although we are using a simple example of galaxy evolution, it is encouraging that the SD scenario reproduces a clear correlation which agrees remarkably well with observations. On the other hand, in the Original model, the SNIa production is shifted to later times compared to the SD scenario, beginning at $\sim 1 \times 10^{8} \mathrm{yrs}$. This excludes the so-called prompt type Ia SNe, that are indeed observed, additionally this model fails at reproducing the slope of the observed correlation. In a forthcoming paper we will explore different SNIa progenitor scenarios applying galaxy formation models to understand the nature of the SNIa progenitors.

\section{Acknowledgments}

NJ warmly thanks the organizers of the IAU 309 Symposium and the financial aid received. NJ acknowledges support from the European Commission's Framework Programme 7, through the Marie Curie International Research Staff Exchange Scheme LACEGAL (PIRSES-GA-2010-269264). This work has been partially supported by "Proyecto Interno" of UNAB (Chile), PIP Conicet-2009/0305 and PICT Racies-2011/959 (Argentina). Simulations were run in Fenix Cluster (IAFE, CONICET-UBA) and Hal Cluster (UNC).

\section{References}

Bensby, T., Yee, J. C., Feltzing, S., et al. 2013, A\&A, 549, A147

Greggio, L. 2010, MNRAS, 406, 22

Jiménez, N., Cora, S. A., Bassino, L. P., Tecce, T. E., \& Smith Castelli, A. V. 2011, MNRAS, 417,785

Kobayashi, C. 2004, MNRAS, 347, 740

Lecureur, A., Hill, V., Zoccali, M., et al. 2007, A\&A, 465, 799

Li, W., Chornock, R., Leaman, J., et al. 2011, MNRAS, 412, 1473

Mannucci, F., Della Valle, M., \& Panagia, N. 2006, MNRAS, 370, 773

Mannucci, F., Della Valle, M., Panagia, N., et al. 2005, A\&SA, 433, 807

Matteucci, F. \& Greggio, L. 1986, A\& $A, 154,279$

Matteucci, F. \& Recchi, S. 2001, ApJ, 558, 351

Scannapieco, C., Tissera, P. B., White, S. D. M., \& Springel, V. 2005, MNRAS, 364, 552

Scannapieco, C., Tissera, P. B., White, S. D. M., \& Springel, V. 2006, MNRAS, 371, 1125

Smith, M., Nichol, R. C., Dilday, B., et al. 2012, ApJ, 755, 61

Springel, V. 2005, MNRAS, 364, 1105

Sullivan, M., Le Borgne, D., Pritchet, C. J., Hodsman, A., \& Neill. 2006, ApJ, 648, 868

Tinsley, B. M. 1979, ApJ, 229, 1046

Tissera, P. B., Beers, T. C., Carollo, D., \& Scannapieco, C. 2014, MNRAS, 439, 3128

Tissera, P. B., Scannapieco, C., Beers, T. C., \& Carollo, D. 2013, MNRAS, 432, 3391

Woosley, S. E. \& Weaver, T. A. 1995, ApJS, 101, 181 